\title{
Inference of Reaction Rate Parameters based on Summary Statistics from Experiments
}

\author{
M. Khalil,,${ }^{a, *}$ K. Chowdhary, ${ }^{a}$ C. Safta,${ }^{a}$ K. Sargsyan,${ }^{a}$ H. N. Najm ${ }^{a}$ \\ ${ }^{a}$ Combustion Research Facility, Sandia National Laboratories, Livermore, CA 94551, USA \\ * Corresponding author: mkhalil@sandia.gov
}

\begin{abstract}
We present the results of an application of Bayesian inference and maximum entropy methods for the estimation of the joint probability density for the Arrhenius rate parameters of the rate coefficient of the $\mathrm{H}_{2} / \mathrm{O}_{2}$-mechanism chain branching reaction $\mathrm{H}+\mathrm{O}_{2} \rightarrow \mathrm{OH}+\mathrm{O}$. Available published data is summary statistics in terms of nominal values and error bars of the rate coefficient of this reaction at a number of temperature values obtained from shock-tube experiments. Our approach relies on generating data, in this case $\mathrm{OH}$ concentration profiles, consistent with the given summary statistics, using Approximate Bayesian Computation methods and a Markov Chain Monte Carlo procedure. The approach permits the forward propagation of parametric uncertainty through the computational model in a manner that is consistent with the published statistics. A consensus joint posterior on the parameters is obtained by pooling the posterior parameter densities given each consistent data set. To expedite this process, we construct efficient surrogates for the $\mathrm{OH}$ concentration using a combination of Padé and polynomial approximants. These surrogate models adequately represent forward model observables and their dependence on input parameters and are computationally efficient to allow their use in the Bayesian inference procedure. We also utilize Gauss-Hermite quadrature with Gaussian proposal probability density functions for moment computation resulting in orders of magnitude speedup in data likelihood evaluation. Despite the strong non-linearity in the model, the consistent data sets all result in nearly Gaussian conditional parameter probability density functions. The technique also accounts for nuisance parameters in the form of Arrhenius parameters of other rate coefficients with prescribed uncertainty. The resulting pooled parameter probability density function is propagated through stoichiometric hydrogen-air auto-ignition computations to illustrate the need to account for correlation among the Arrhenius rate parameters of one reaction and across rate parameters of different reactions.
\end{abstract}

Keywords: Uncertainty Quantification, Bayesian Inference, Reaction Rates, Arrhenius Parameters, Shock Tube Experiments

(C) 2016. This manuscript version is made available under the Elsevier user license http://www.elsevier.com/open-access/userlicense/1.0/ 


\section{Introduction}

Chemical kinetic models for hydrocarbon fuels commonly involve $\mathcal{O}\left(10^{2}-10^{4}\right)$ elementary reactions. Experimentalists have traditionally reported nominal elementary reaction rates with uncertainty estimates in the form of error bars or uncertainty factors assigned to rate coefficients, with the original experimental data not published. In order for modelers to make reliable computational predictions with these chemical kinetic models, the various sources of uncertainty including reaction rates must be characterized accurately and then propagated using uncertainty quantification (UQ) methods (see [1-3] for a review). This issue has received increased attention from the modeling community in a variety of contexts in recent years. Frenklach [4] discusses the role of uncertainty analysis and reduction in the context of model development. You et al. [5] developed an approach for the calibration of combustion models by constraining the optimization to both parameter uncertainties and uncertainties in experimental data. Nagy and Turányi [6] developed a method for characterizing the uncertainty in the Arrhenius parameters from the information available in kinetic databases. Sheen et al. [7] calibrated a model for the oxidation of unsaturated hydrocarbons against a range of measurements of the $\mathrm{H}$ atom attack process in single-pulse shock tubes using the method of uncertainty minimization by polynomial chaos expansion. Xin et al. [8] used the same methodology in providing reduced kinetic models for n-butane and iso-butane. Goldsmith et al. [9] perform global sensitivity analysis for the phenomenological rate constants for $\mathrm{O}_{2}+\mathrm{n}$-propyl. Tomlin [10] provides an overview of the available methodologies for local and global sensitivity and uncertainty analysis for kinetic mechanisms. Hakim et al. [11] apply Bayesian inference in the calibration of simple Arrhenius mechanisms for simulation of Diesel engine combustion with uncertainties.

Often, modelers interpret the uncertainty factors in the reaction rates, as reported by experimentalists, as uncertainties in the pre-exponential factor of the Arrhenius rate expression. This approach is limiting, as it ignores uncertainty in the temperature exponent and activation energy, resulting in uncertainties in reaction rates that are constant in temperature. As an improvement on this approach, Nagy and Turányi [6] proposed a procedure for fitting a truncated multivariate normal distribution for the Arrhenius parameters to temperature-dependent uncertainty factors. The method, however, considers data only in the form of uncertainty factors on the rate coefficients in providing a joint Arrhenius parameter PDF but does not consider the fit model (reaction mechanism) used by the experimentalists, the detailed experimental condi- 
tions, or the statistical representation of measurement uncertainty to further inform the joint posterior parameter PDF. Turányi et al. [12] proposed a framework for the optimization of Arrhenius parameters of rate coefficients for multiple reactions using direct (e.g. rate coefficient) and indirect (e.g. ignition delay and flame speed) data and providing a covariance matrix to characterize the joint uncertainty of these parameters. The method provides a joint Gaussian distribution on the unknown Arrhenius parameters and was applied for joint characterization of the Arrhenius parameters of the rate coefficients of $\mathrm{H}+\mathrm{O}_{2} \rightarrow \mathrm{OH}+\mathrm{O}$ and $\mathrm{H}+\mathrm{O} 2+\mathrm{M} \rightarrow \mathrm{HO} 2+\mathrm{M}$. The method was recently utilized to provide an optimized hydrogen combustion mechanism [13]. The technique used in this investigation is fully Bayesian in that no Gaussian approximation is assumed for the Arrhenius parameters. It also considers the fit model as well as experimental conditions used by the experimentalists in obtaining the direct data on rate coefficient, which in tandem greatly inform the joint distribution (e.g. correlation) of the Arrhenius parameters. Furthermore, the proposed method incorporates prescribed marginal uncertainty in the Arrhenius parameters of rate coefficients for other reactions, acting as nuisance parameters in the inference process.

For a probabilistic forward UQ analysis, a joint PDF on the Arrhenius parameters is needed. Bayesian inference methods [14] can be used for estimating these joint PDFs given experimental data. For example, Miki et al. [15] applied Bayesian inference to quantify the uncertainty in the rate coefficient of $\mathrm{H}+\mathrm{O}_{2} \rightarrow \mathrm{OH}+\mathrm{O}$ using shocktube experimental data. Mosbachet al. [16] applied Bayesian inference to estimate the Arrhenius parameters in a chemical kinetic mechanism for n-propylbenzene oxidation using shock-tube experimental data. In the context of experimental chemical kinetics in combustion, however, the experimental data used in obtaining the nominal reaction rates and uncertainty factors is typically unavailable. The only information available that could inform a posterior PDF on the parameters are the published nominals and uncertainty factors, which can be regarded as summary statistics of the missing data. Berry et al. [17] developed a framework capable of handling such problems. The method, dubbed Data Free Inference (DFI), uses the maximum entropy (MaxEnt) principle to enforce the given summary statistics and relevant experimental parameters using Approximate Bayesian Computation (ABC) [18]. It provides a joint posterior density on both data and model parameters consistent with the published summary statistics. The implicit marginalization of this joint posterior provides the sought-after posterior on the parameters of interest. The resulting construction involves the solution of a nested pair of Bayesian inference problems, an outer one on the data-space and an 
inner one on the parameters. Najm et al. [19] applied the technique to infer uncertain model parameters for a global 1-step chemical model of methane-air ignition from synthetic data. Chowdhary and Najm [20] demonstrated the technique for processed data products in general, specifically targeting summary statistics on model outputs.

In this investigation we apply DFI to estimate the joint PDF on the Arrhenius parameters for the rate coefficient, $k$, of the $\mathrm{H}_{2} / \mathrm{O}_{2}$ mechanism chain branching reaction $\mathrm{H}+\mathrm{O}_{2} \rightarrow \mathrm{OH}+\mathrm{O}$. This is one of the most important elementary reactions in combustion, being an essential component of the $\mathrm{H}_{2} / \mathrm{O}_{2}$ reaction system, which contains a number of reactions whose rate constants are among the most sensitive for combustion predictions for all hydrocarbon or oxygenated fuels [21]. We have chosen this reaction system as a test bed due to its importance to a variety of applications in energy conversion. There have been many published experimental measurements of this reaction. We will focus on the published data, in the form of summary statistics in terms of nominal values and error bars of $k$ at a number of temperature values, obtained from shock-tube experiments performed by Masten et al. [22].

The original method developed by Berry et al. [17] to infer model parameters from summary statistics involves a two level random walk procedure, one being on the data space and the other on the parameter space. The algorithm proposes data sets, accepting those that, when employed for fitting the given model parameters, provide nominal values and bounds that are consistent with those published. The resulting consistent data sets subsequently provide a pooled/averaged joint PDF on the parameters using Bayesian inference through a Markov Chain Monte Carlo (MCMC) sampling technique [23]. The algorithm is computationally taxing due to the nested randomwalk/MCMC structure, especially given the high dimensionality of the outer/data chain in this case. We propose a modification, involving the use of Gauss-Hermite quadrature [24] for parameter inference, resulting in orders of magnitude speedup in data likelihood evaluation. This approach is useful when the parameter vector is lowdimensional, which is the case here, and when the posterior parameter PDF is nearly Gaussian, which we also find to be true here. To further expedite the process, we construct hybrid Padé and polynomial approximants as surrogates for the missing $\mathrm{OH}$ concentration profiles.

Masten et al. [22] inferred $k$ with the shock-tube experimental data with an assigned uncertainty of $40 \%$ to the rate coefficient $k_{2}$ of the reaction $\mathrm{OH}+\mathrm{H}_{2} \rightarrow \mathrm{H}_{2} \mathrm{O}+\mathrm{H}$. For consistency, we model its pre-exponential $A_{2}$ as a prescribed log-normal random variable that is employed as a nuisance parameter, not inferred along with the Arrhenius 
parameters of $k$.

Aside from reporting the above algorithmic advances, including means of accelerating the algorithm, and the inclusion of nuisance parameters, this paper demonstrates the application of the method in a highly relevant context, and illustrates the need for proper accounting for Arrhenius rate coefficient correlations in making ignition predictions with uncertainty.

The rest of the text is organized as follows. Section 2 summarizes the results from the shock tube experiments performed by Masten et al. [22] to be used in this investigation. Section 3 presents an overview of the algorithm. The method is then applied to the available data in Section 4. Finally, Section 5 presents our conclusions.

\section{Data From Shock Tube Experiments}

Masten et al. [22] reported results of shock tube experiments in the form of reaction rate coefficients, $k$, for the reaction $\mathrm{H}+\mathrm{O}_{2} \rightarrow \mathrm{OH}+\mathrm{O}$ at various initial temperatures and mixtures. Mixtures of hydrogen and oxygen diluted with argon were heated by both incident and reflected shock waves in the temperature range 1450-3370 K. Results included uncertainties associated with the reported values. We will assimilate all data points (from 30 experiments) reported except for the highest temperature data point at $T=3370 \mathrm{~K}$. We do not use this last point because the uncertainty of the rate coefficient of the reaction $\mathrm{H}_{2}+\mathrm{M} \rightarrow 2 \mathrm{H}+\mathrm{M}$, which [22] accounts for, but we don't, is in fact significant for this one temperature condition. This data point is excluded from the analysis in favor of reducing the number of nuisance parameters to be considered and thus reducing the computational complexity of the analysis. We believe that the exclusion of this data point does not significantly alter the results obtained since (a) the majority of data points are accounted for (29 out of 30) and (b) the uncertainty associated with the excluded data point is twice that of the other data points.

The total uncertainty budget in the rate $k$ is due to the noise in (a) the assimilated $\mathrm{OH}$ profiles and (b) the nuisance-parameter uncertainty in the rate coefficient $k_{2}$ of the reaction $\mathrm{OH}+\mathrm{H}_{2} \rightarrow \mathrm{H}_{2} \mathrm{O}+\mathrm{H}$. An uncertainty factor of 1.06 (i.e. $6 \%$ uncertainty) in the $k$ values is reported at all temperatures due to the two aforementioned sources of uncertainty [25]. The uncertainty factor, $U F=k_{\text {nom }} / k_{\min }=k_{\max } / k_{\text {nom }}$, is widely used to report uncertainty in rate coefficients with $k_{\text {nom }}$ being the recommended value, and $k_{\min }$ and $k_{\max }$ the possible extremes, which we interpret to be 2 standard deviations from the recommended value on a logarithmic scale. Thus, the uncertainty factor and 
the standard deviation of the logarithm of the rate coefficient, $(\ln k)_{\mathrm{sd}}$, relate to each other using $(\ln k)_{\mathrm{sd}}=(\ln U F) / 2$. The $\mathrm{OH}$ time traces used in fitting were determined to be most sensitive to $k$, but also sensitive to a lesser degree to the $k_{2}$ rate coefficient of the reaction $\mathrm{OH}+\mathrm{H}_{2} \rightarrow \mathrm{H}_{2} \mathrm{O}+\mathrm{H}$. Given the stated $k_{2}$ uncertainty of $40 \%$ [22], we model the associated pre-exponential factor $A_{2}$ as a log-normal random variable (Gaussian in log-scale), while assigning nominal values to the corresponding temperature exponent and activation energy. The pre-exponential factor $A_{2}$ is not inferred in [22] along with $k$ and thus we will infer $k$ while treating $A_{2}$ as a nuisance parameter for consistency. For brevity, we direct the reader to [22] for the full reaction mechanism used herein. It is noteworthy to mention that the rate coefficient $k$ was determined in [22] by fitting the region of rapid $\mathrm{OH}$ growth, being the region most sensitive to $k$, and, to a lesser extent, $k_{2}$. Thus the missing data is of the form of $\mathrm{OH}$ time profiles in which rapid $\mathrm{OH}$ growth is observed.

We assume that the missing data was captured discretely in time at a constant sampling rate, which is a priori unknown. What is known is that the $\mathrm{OH}$ data used in calibration is in the region of rapid $\mathrm{OH}$ growth. We determine the corresponding time intervals at each temperature by performing forward simulations of the numerical model at the nominal reaction rates in [22]. Thus the only remaining variable is $N$, the number of time instances at which $\mathrm{OH}$ concentration is available. To reflect the uncertainty in $\mathrm{OH}$ concentration levels obtained from shock tube experiments, we assume a Gaussian additive noise term to represent measurement error as in

$$
\left[\mathrm{OH}_{d}\right]_{i, j}=\left[\mathrm{OH}_{m}\right]_{i, j}+\sigma \epsilon_{i, j}
$$

where $\epsilon_{i, j}$ are independent and identically distributed (i.i.d.) standard normal variables, $\sigma$ is the standard deviation of the noise, subscript $i=1, \ldots, N$ denotes the time instance at which data is acquired, subscript $j=1, \ldots, 29$ denotes the shock tube experiment number with 29 experiments in total, $\left[\mathrm{OH}_{m}\right]_{i, j}$ is the model generated $\mathrm{OH}$ concentration at time instance $t_{i}$ for experiment $j$, and $\left[\mathrm{OH}_{d}\right]_{i, j}$ is the corresponding measurement data.

\section{Algorithm}

The algorithm used herein is presented in full detail in [17]. Here we provide an outline for brevity. We take as inputs the specification of the fit model (ignition using the reaction mechanism from [22]), the instrument noise model, the presumed 
number of missing data points, the initial conditions of each of the 29 experiments, nominal $k$ values, and associated standard deviations. We explore the missing data space using an MCMC procedure. For each proposed data set, we infer the unknown Arrhenius parameters of rate $k$ as in $k=A T^{n} \exp (-E / R T)$, where $R$ is the universal gas constant, $T$ is the temperature, $E$ is the activation energy, and $A$ is the preexponential constant. The inference is carried out with the pre-exponential factor $A_{2}$ of the rate coefficient of reaction $\mathrm{OH}+\mathrm{H}_{2} \rightarrow \mathrm{H}_{2} \mathrm{O}+\mathrm{H}$ as a nuisance parameter and this process involves constructing the joint posterior $\mathrm{PDF}$ on $\left(\ln A, n, E, \ln A_{2}\right)$ with a prescribed normal marginal PDF for $\ln A_{2}$. The procedure relies on error model Eq. (1) with $\sigma$ an unknown hyper-parameter. The inference of the logarithms of the pre-exponential factors and $\sigma$, as opposed to the parameters themselves, enforces their positivity.

For the current investigation, the summary statistics, denoted by $I$, comprised of nominal values for $\ln k$ at each experiment denoted by $\ln k_{\text {nom,j }}, j=1, \ldots, 29$ as published in [22] and associated standard deviations of 0.02913 denoted by $(\ln k)_{\mathrm{sd}, j}, j=$ $1, \ldots, 29$ equivalent to an uncertainty factor of $6 \%$ in $k$. Let us denote the data vector for the MCMC (data) chain by $\boldsymbol{z}$ and the parameter vector to be inferred by $\lambda$, i.e. $\lambda=(\theta, \phi)$, with $\theta=(\ln A, n, E, \ln \sigma)$ and $\phi=\ln A_{2}$. The parameters are divided into two groups, $\theta$ and $\phi$, with the latter denoting the set of nuisance parameters having a prescribed PDF.

The MCMC (data) chain will solve the Bayesian inference problem

$$
p(\boldsymbol{z} \mid I) \propto p(I \mid \boldsymbol{z}) \pi(\boldsymbol{z})
$$

where $\boldsymbol{z}$ is the data vector, $p(\boldsymbol{z} \mid I)$ is the corresponding posterior PDF, $p(I \mid \boldsymbol{z})$ the likelihood function, and $\pi(\boldsymbol{z})$ the prior PDF. For every proposed $z$, an inner inference problem is solved over the parameter space. Gauss-Hermite quadrature will be used to extract moments from the posterior PDF on the parameters:

$$
p(\lambda \mid \boldsymbol{z})=p(\theta, \phi \mid \boldsymbol{z})=\frac{p(\boldsymbol{z} \mid \theta, \phi) p(\theta \mid \phi)}{p(\boldsymbol{z} \mid \phi)} q(\phi),
$$

in which $q(\phi)$ is the prescribed marginal PDF over the nuisance parameter $\phi=\ln A_{2}$, $p(\boldsymbol{z} \mid \theta, \phi)$ is the likelihood function of the data given the parameters, $p(\theta \mid \phi)$ is the prior PDF for $\theta$ conditional on $\phi$, and $p(\boldsymbol{z} \mid \phi)$ is a normalization constant that depends on $\phi$. The $\mathrm{ABC}$ data likelihood, aiming to provide consistent data sets with the given 
reaction rate statistics, is given by:

$$
p(I \mid \boldsymbol{z}) \propto \prod_{j=1}^{29} f_{\delta}\left(\mu_{j} \mid \ln k_{\mathrm{nom}, j}\right) f_{\delta}\left(\sigma_{j} \mid(\ln k)_{\mathrm{sd}, j}\right)
$$

where $\mu_{j}$ and $\sigma_{j}$ are the pushed-forward mean and standard deviation of $\ln k$, given by

$$
\begin{aligned}
& \mu_{j}=\int\left(\theta_{1}+\theta_{2} T_{j}-\theta_{3} / R T_{j}\right) p(\theta, \phi \mid \boldsymbol{z}) d \theta d \phi, \\
& \sigma_{j}^{2}=\int\left(\theta_{1}+\theta_{2} T_{j}-\theta_{3} / R T_{j}-\mu_{j}\right)^{2} p(\theta, \phi \mid \boldsymbol{z}) d \theta d \phi,
\end{aligned}
$$

where $T_{j}$ is the initial temperature for experiment $j$. Despite the strong non-linearity in the model, the consistent data sets all result in nearly Gaussian parameter posteriors. Thus, Gauss-Hermite quadrature [24] is used to approximate the above integrals. The quadrature points are obtained as a 5-D tensor product of a 3-point 1-D Gauss-Hermite quadrature rule, resulting in a total of 243 forward model runs for a given realization of the data vector $\boldsymbol{z}$ to estimate the above integrals. This results in orders of magnitude speedup in data likelihood evaluation over the use of Monte Carlo sampling techniques (such as MCMC).

In Eq. (4) the function $f_{\delta}$ is a normalized variate of the Gaussian kernel function given by

$$
f_{\delta}(x \mid y)=\exp \left\{-\delta\left(\frac{x-y}{y}\right)^{2}\right\}
$$

and $\delta$ is a positive constant that dictates a requisite level of consistency of the proposed data sets with the published statistics. The larger the $\delta$ value used the closer is the consistency required between the proposed data sets and the given statistics.

For each proposed $\boldsymbol{z}$ in the MCMC chain, Gauss-Hermite quadrature of Eqs. (5)-(6) provides statistics for the logarithm of the rate coefficients. These then provide the likelihood $p(I \mid \boldsymbol{z})$, and subsequently the posterior $p(\boldsymbol{z} \mid I)$, of the data set which enables the MCMC algorithm to accept or reject the proposed data set.

We assume that $N$ data points are used in representing the missing $\mathrm{OH}$ profile $\left(\left[\mathrm{OH}_{d}\right]_{i, j}, i=0, \ldots, N-1\right)$ for each of the 29 experiments, with $j$ denoting the experiment number and $i$ the predetermined time instance $t_{i}$. The MCMC chain is thus $(29 \times N)$-dimensional, with the state vector $\boldsymbol{z}$ given by $\boldsymbol{z}=\left(z_{1}, \ldots, z_{29}\right)$, where $z_{j}=\left(\left[\mathrm{OH}_{d}\right]_{1, j}, \ldots,\left[\mathrm{OH}_{d}\right]_{N, j}\right)$ is the proposed data set (OH profile) for shock-tube experiment $j$. 
The likelihood function for parameters $p(\theta, \phi \mid z)$ is constructed using the above assumption of Gaussian additive data noise in Eq. (1). To further expedite the computation of the integrals Eqs. (5)-(6), we use hybrid Padé and polynomial approximants as surrogates for the model-predicted $\mathrm{OH}$ concentration profiles in terms of time, $k$ and $k_{2}$ (details excluded for brevity). To construct these surrogates, we compute $\mathrm{OH}$ concentration profiles using constant pressure ignition computations employing the TChem [26] thermochemical library and the CVODE [27] time integration library.

The MCMC data chain provides a number of consistent data sets, each with a corresponding consistent posterior on the model parameter vector $\lambda$. The use of logarithmic pooling (see [17] for details) results in an averaged posterior on the parameters. This pooled posterior represents the best state of knowledge given the published statistics. Algorithm DFI outlines the method and the known and unknown parameters.x

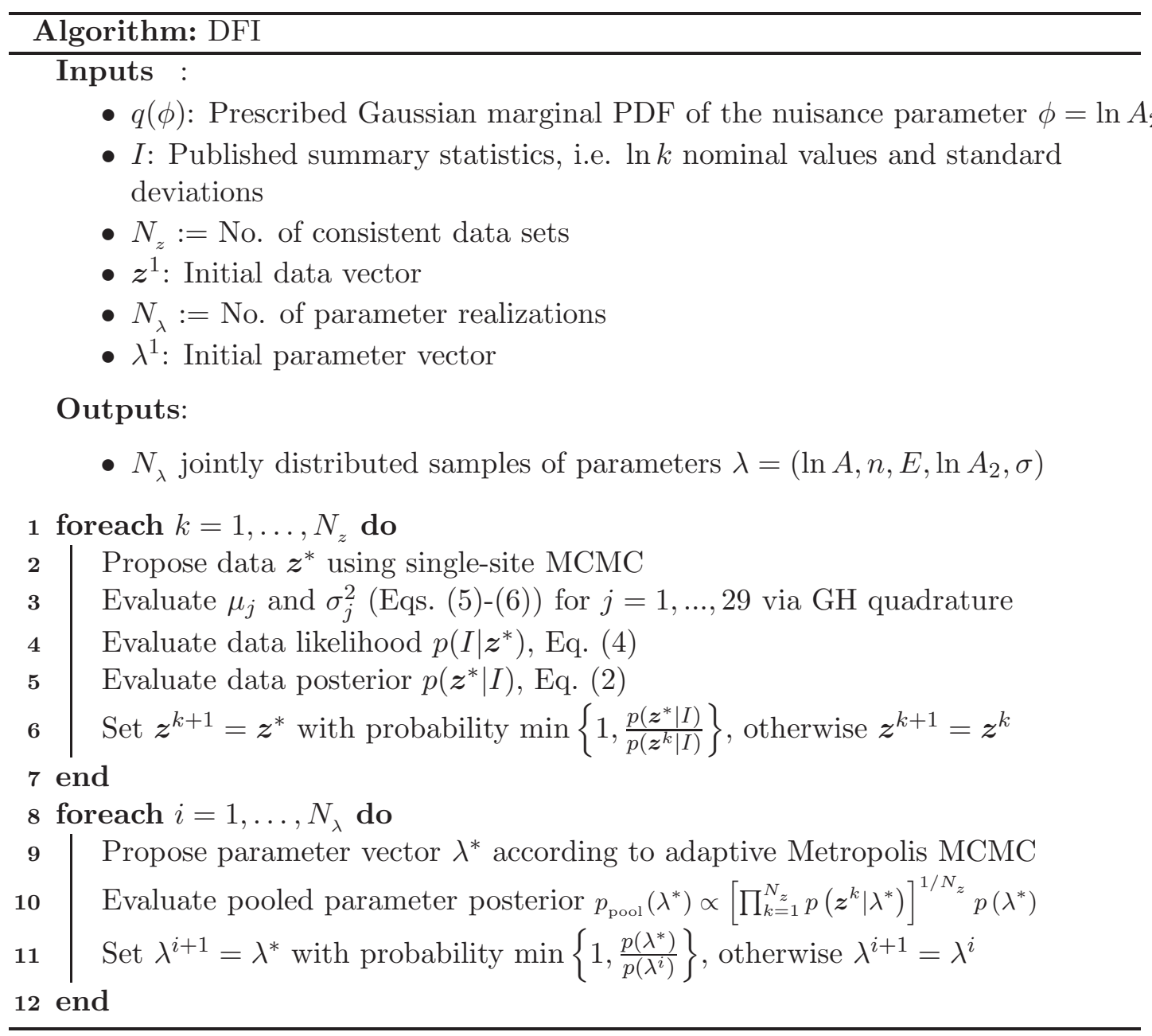




\section{Application to statistics from shock-tube experiments}

We apply the above technique to estimate the joint PDF of the Arrhenius parameters for the rate coefficient, $k$, of the reaction $\mathrm{H}+\mathrm{O}_{2} \rightarrow \mathrm{OH}+\mathrm{O}$ using published statistics from Masten et al. [22] as described above. We infer the unknown data and parameter vectors while assuming that $N=10$ data points for each experiment sufficiently captures the $\mathrm{OH}$ profile characteristics for parameter inference. While one can represent $N$ as an uncertain quantity with a specified PDF, and proceed by marginalizing over it, we have found that the results vary little with an increase of $N$ beyond 10. For the consistency check we use $\delta=500$ which results in relatively small scatter of statistics from proposed data sets.

The initial condition for the MCMC chain on the data space is obtained by contaminating computed ignition $\mathrm{OH}$ profiles, obtained using the nominal parameter values given by Masten et al., with additive i.i.d. Gaussian noise. The standard deviation of the noise process is chosen so as to maximize the data posterior in Eq. (2). Once that initial data set is obtained, the data vector is sampled using the conventional single-site Metropolis-Hastings MCMC algorithm with a Gaussian proposal PDF of pre-specified constant variance chosen to result in approximately $25 \%$ acceptance ratio. Ten $10^{4}$-step long chains are run in parallel beyond the associated initial burn-in period resulting in a total of $10^{5}$ consistent data sets. Using these, we compute a pooled parameter posterior PDF using an MCMC chain on the parameter space. A total of $10^{6}$ realizations of the parameter vector are obtained, from which normalized histograms are computed as estimates of selected $1 \mathrm{D}$ and $2 \mathrm{D}$ marginal PDFs, as shown in Figs. 1 and 2 , respectively. The evidence, or normalization constant, $p(\boldsymbol{z} \mid \phi)$ was computed on a uniform mesh in $\phi$ using 4-D Gauss-Hermite quadrature, and subsequently linearly interpolated for different values of $\phi=\ln A_{2}$.

The strong Gaussian trend in the posterior parameter PDF is evident from the selected 1D and 2D marginal PDFs in Figs. 1-2, justifying the use of Gaussian proposals. Included in the marginal PDF plots are the nominal values reported by Masten et al. [22], which are probable under the resulting joint parameter PDF. Note that Masten et al. [22] performed the calibration with the constraint that the temperature exponent $n$ is zero. The 2D marginal PDFs illustrate strong correlations among the three Arrhenius parameters of the rate coefficient $k$. The pre-exponential factor $A_{2}$ of the rate coefficient of reaction $\mathrm{OH}+\mathrm{H}_{2} \rightarrow \mathrm{H}_{2} \mathrm{O}+\mathrm{H}$ exhibits weak correlation, in logarithmic space, with the other pre-exponential factor $A$. It is important to high- 
a)

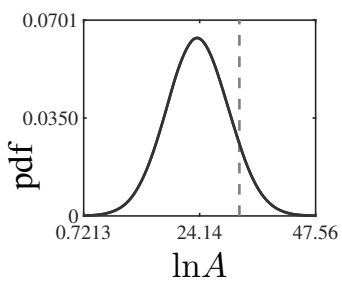

c)

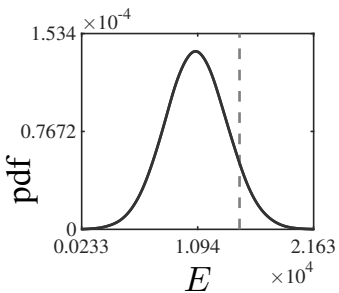

b)

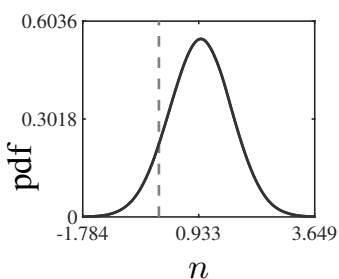

d)

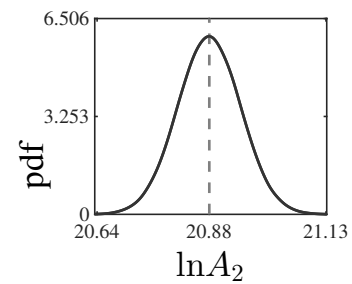

Fig. 1: Selected 1D marginal parameter PDFs. Dashed lines denote nominal values reported by Masten et al. $[22]$

a)

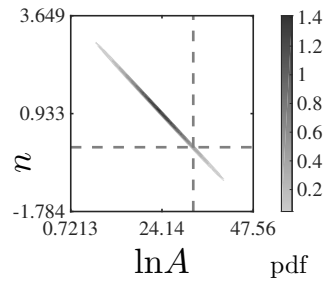

c)

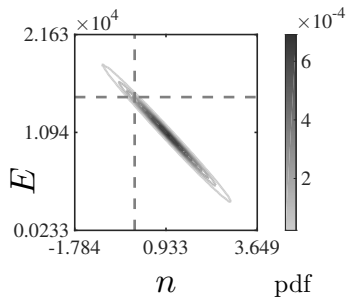

b)

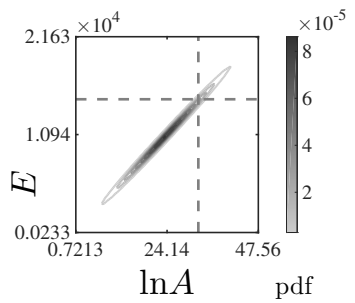

d)

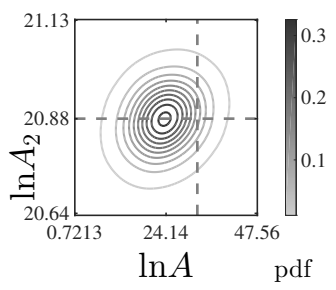

Fig. 2: Selected 2D marginal parameter PDFs. Dashed lines denote nominal values reported by Masten et al. $[22]$

light the nonzero correlation among Arrhenius parameters for one rate coefficient as well as across rate coefficients. The discovery of this joint correlated PDF on these parameters, which, by construction, is consistent with the computed ignition behavior and published experimental data, is a key objective of this work. The strong degree of correlation should raise doubts as to the efficacy of ignoring such correlations, and proceeding with an independence assumption on uncertain model parameters. We will next propagate the full joint PDF of the Arrhenius rate parameters through ignition computations using the same MCMC samples and examine the effect of ignoring the correlations between the various parameters while making numerical predictions under 
uncertainty.

We illustrate the need for propagating the full joint PDF of the Arrhenius parameters through ignition computations for three cases: (a) The three Arrhenius parameters of $k$, i.e. $\ln A, n$ and $E$, and the pre-exponential $\ln A_{2}$ are jointly distributed according to the pooled parameter posterior PDF obtained in this investigation, (b) $n$ and $E$ are assigned maximum-a-posteriori (MAP) estimates and the two pre-exponential factors $\ln A$ and $\ln A_{2}$ are assumed to be independently distributed according to the marginal pooled parameter PDF conditional on the chosen values of $n$ and $E$, and (c) $E$ and $A$ are independent Gaussian random variables with mean values and associated standard deviations as reported in Masten et al. [22] with $n$ fixed at zero and $\ln A_{2}$ treated as an independent random variable with a Gaussian PDF reflecting the uncertainty factor found in Masten et al. [22]. We first examine the predicted rate coefficient $k$ with the three scenarios described above, with the results for case (a) shown in Fig. 3 and for cases (b) and (c) presented in Fig. 4. We can observe that the median estimate for the rate coefficient as a function of the inverse of the temperature does not vary significantly between the three cases investigated. The confidence interval, however, is overestimated using cases (b) and (c) in the range of the data $\left(3.5<10^{4} / T<7\right)$, whereas the confidence interval obtained using the full pooled parameter PDF (case (a)) is generally in good agreement with the statistics published by Masten et al. [22]. Moreover, we see that both cases (a) and (c) exhibit temperature-dependent uncertainty, as expected, with stronger growth of uncertainty in case (a) as we move outside the data range. Clearly, strong correlations have significant consequences, and should not be ignored.

We next use the above PDFs for prediction with uncertainty. We examine ignition delay time PDFs for stoichiometric hydrogen-air auto-ignition at initial temperature of $1000 \mathrm{~K}$ and (constant) atmospheric pressure for cases (a-c), as shown in Fig. 5. We define the onset of ignition to be the time instance at which the temperature reaches $500 \mathrm{~K}$ above initial value. We observe that the predicted ignition delay PDF varies greatly for the three cases. The pooled parameter PDF results in the greatest uncertainty (greatest variance) in ignition delay time while providing the lowest (mean) estimate. It is important to recall that the parameter estimation was performed with data in the $1450 \mathrm{~K}$ to $2900 \mathrm{~K}$ range (Fig. 3), while the initial temperature here is $1000 \mathrm{~K}$. We are therefore extrapolating the rate coefficient in predicting the ignition delay time. Figs. 3-4 illustrate that the greatest uncertainty in extrapolating the rate coefficient to $T=1000 \mathrm{~K}\left(10^{4} / T=10\right)$ is observed for case (a), resulting in the greatest uncertainties 


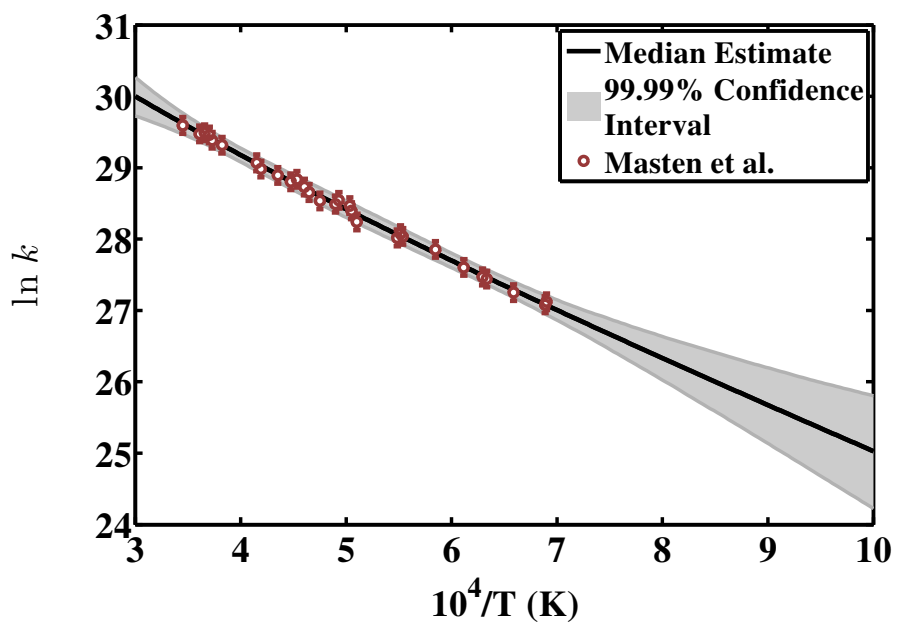

Fig. 3: Arrhenius plot of rate coefficient $k$ illustrating the median estimate (black curve) and corresponding 99\% confidence interval (gray area). Estimates obtained from experiments performed by Masten et al. [22] are shown in circles with associated error bars (scaled to represent $99 \%$ confidence intervals).

in ignition time for an initial temperature of $1000 \mathrm{~K}$. This growth of uncertainty in extrapolative predictions for case (a) in comparison to the other cases is to be expected, as more uncertain parameters are considered. A more detailed/comprehensive model provides more flexibility in fitting the data within the associated range, as seen in Fig 3. However, it is also more prone to over-fitting the data. One must exercise caution when using highly-detailed models for extrapolation, as they can result in divergent confidence intervals.

We also extract the median temperature profiles and associated $99 \%$ confidence intervals as shown in Fig. 6. We see that, again, case (a) results in the greatest uncertainty out of the three cases examined. Similar trends were observed for H, O, and $\mathrm{OH}$ radical concentration profiles, i.e. greatest uncertainty in these profiles was observed for case (a), with results not included for brevity. This growth of uncertainty in extrapolative predictions for case (a) in comparison to the other cases is to be expected as more uncertain parameters are considered. A more comprehensive model provides more flexibility in fitting the data within the associated range, as seen in Fig. 3. At the same time a more comprehensive model is more prone to over fitting the data and thus one must exercise caution when using such model in making extrapolations as it often results in divergent confidence intervals as highlighted by the reviewer.

We also carried out ignition simulations for the three cases at a higher initial temperature of $1500 \mathrm{~K}$ with the resulting ignition delay time PDFs shown in Fig. 7 
a)

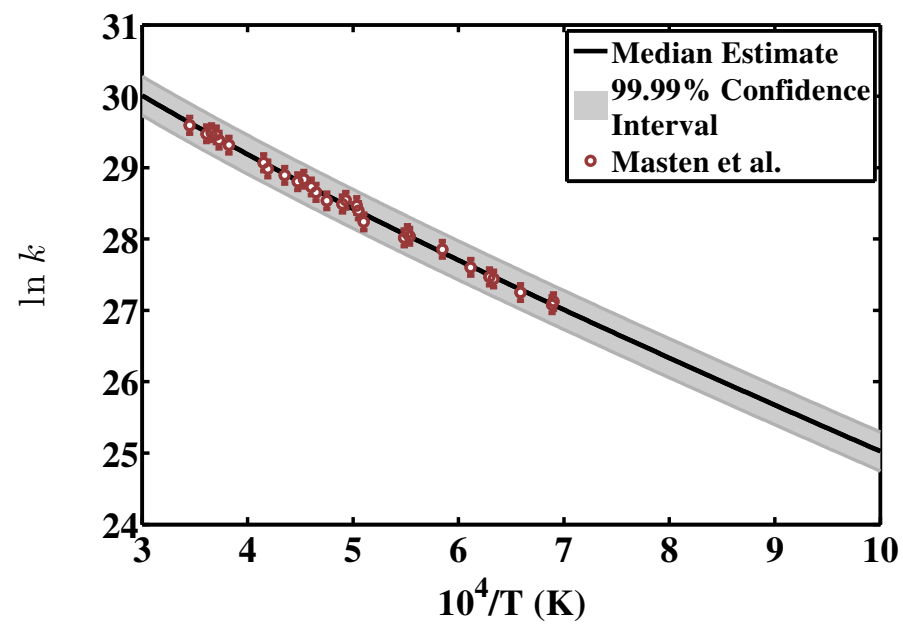

b)

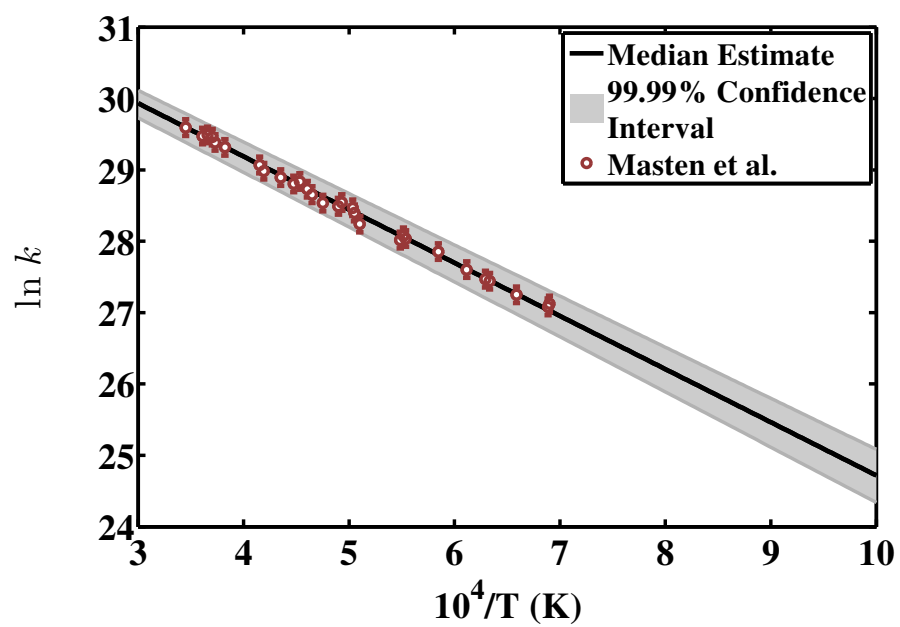

Fig. 4: Arrhenius plot of rate coefficient $k$ illustrating the median estimate (black curve) and corresponding 99\% confidence interval (gray area): (a) $n$ and $E$ are assigned maximum-a-posteriori (MAP) estimates and $\ln A$ is distributed according to the pooled parameter posterior PDF conditional on $n$ and $E$; (b) $E$ and $A$ are independent Gaussian random variables with mean values and standard deviations as reported in Masten et al. [22] with $n$ fixed at zero. Estimates obtained from experiments performed by Masten et al. [22] are shown in circles with associated error bars (scaled to represent 99\% confidence intervals).

and the temperature profiles in Fig. 8. In this context, case (a) results in predictions with the least uncertainty out of the three cases. Those results are expected given the more compact confidence interval of the rate coefficient $k$ seen for case (a) for temperature within the data range, as shown in Fig. 3.

\section{Conclusion}




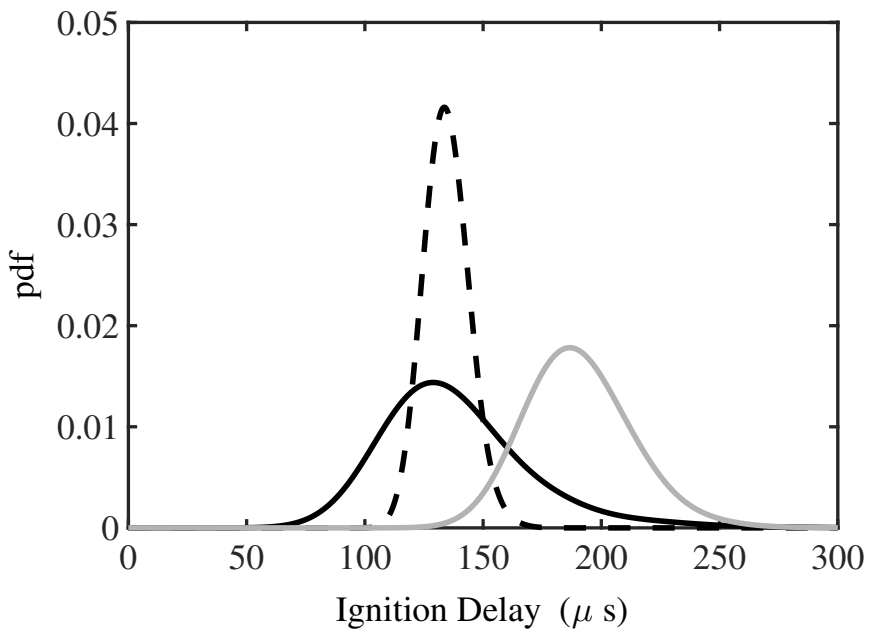

Fig. 5: Ignition delay time PDF for stoichiometric hydrogen-air auto-ignition at initial temperature of $1000 \mathrm{~K}$ and atmospheric pressure with Arrhenius parameters distributed according to case (a) in solid black curve, case (b) in dashed black curve, and case (c) in solid gray curve.

We presented the results of an application of Bayesian inference to the estimation of the joint probability density on the Arrhenius parameters of the reaction $\mathrm{H}+\mathrm{O}_{2} \rightarrow \mathrm{OH}+\mathrm{O}$ in the absence of shock-tube experimental data. Available published results in the form of summary statistics, being nominal values and error bars of the rate coefficient at a number of temperatures, were successfully assimilated to generate consistent $\mathrm{OH}$ concentration profiles, and associated joint posterior densities on the Arrhenius parameters, using a hybrid Markov Chain Monte Carlo and GaussHermite quadrature procedure. The technique used herein utilizes data in the form of uncertainty factors, the fit model (reaction mechanism) used by the experimentalists, the detailed experimental conditions, and the statistical representation of measurement uncertainty to provide a consensus joint posterior on the Arrhenius parameters. The technique also allows us to handle nuisance parameters in the form of Arrhenius parameters of other rate coefficients with prescribed uncertainty. The propagation of the resulting full joint Arrhenius parameter PDF through stoichiometric hydrogenair auto-ignition computations results in different predictions in ignition delay times and temperature profiles in comparison to the cases in which the correlation structure among the Arrhenius parameters is ignored. This argues for the importance of proper accounting for correlation among chemical kinetic model parameters in making informed predictions with uncertainty.

\section{Acknowledgements}


This work was supported by the US Department of Energy (DOE), Office of Basic Energy Sciences (BES) Division of Chemical Sciences, Geosciences, and Biosciences. Sandia National Laboratories is a multiprogram laboratory operated by Sandia Corporation, a Lockheed Martin Company, for the United States Department of Energy under contract DE-AC04-94-AL85000.

\section{References}

[1] H. Najm, Annual Review of Fluid Mechanics 41 (1) (2009) 35-52.

[2] D. Xiu, Journal of Computational Physics 5 (2-4) (2009) 242-272.

[3] H. Wang, D. A. Sheen, Progress in Energy and Combustion Science 47 (2015) $1-31$.

[4] M. Frenklach, Proc. Comb. Inst. 31 (1) (2007) 125-140.

[5] X. You, T. Russi, A. Packard, M. Frenklach, Proceedings of the Combustion Institute 33 (1) (2011) 509-516.

[6] T. Nagy, T. Turányi, Reliability Engineering and System Safety 107 (2012) 29-34.

[7] D. Sheen, C. Rosado-Reyes, W. Tsang, Proc. Comb. Inst. 34 (1) (2013) 527-536.

[8] Y. Xin, D. A. Sheen, H. Wang, C. K. Law, Combustion and Flame 161 (12) (2014) 3031-3039.

[9] C. Goldsmith, A. Tomlin, S. Klippenstein, Proc. Comb. Inst. 34 (1) (2013) 177185.

[10] A. Tomlin, Proc. Comb. Inst. 34 (1) (2013) 159-176.

[11] L. Hakim, G. Lacaze, M. Khalil, H. N. Najm, J. C. Oefelein, Journal of Engineering for Gas Turbines and Power (2016), in press.

[12] T. Turányi, T. Nagy, I. Zsély, M. Cserháti, T. Varga, B. Szabó, I. Sedyó, P. Kiss, A. Zempléni, H. Curran, Int. J. Chem. Kinetics 44 (2012) 284-302.

[13] T. Varga, T. Nagy, C. Olm, I. Zsly, R. Plvlgyi, . Valk, G. Vincze, M. Cserhti, H. Curran, T. Turnyi, Proceedings of the Combustion Institute 35 (1) (2015) 589 $-596$.

[14] D. Sivia, Data Analysis: A Bayesian Tutorial, Oxford Science, 1996.

[15] K. Miki, E. E. Prudencio, S. H. Cheung, G. Terejanu, Combustion and Flame 160 (5) (2013) 861-869.

[16] S. Mosbach, J. H. Hong, G. P. Brownbridge, M. Kraft, S. Gudiyella, K. Brezinsky, International Journal of Chemical Kinetics 46 (7) (2014) 389-404. 
[17] R. Berry, H. Najm, B. Debusschere, H. Adalsteinsson, Y. Marzouk, Journal of Computational Physics 231 (2012) 2180-2198.

[18] M. Beaumont, W. Zhang, D. J. Balding, Genetics 162 (4) (2002) 2025-2035.

[19] H. Najm, R. Berry, C. Safta, K. Sargsyan, B. Debusschere, Int. J. for Uncertainty Quantification 4 (2) (2014) 111-132.

[20] K. Chowdhary, H. Najm, Statistics and Computing 26 (1) (2016) 149-169.

[21] C. Westbrook, F. Dryer, Progress in Energy and Combustion Science 10 (1984) $1-57$.

[22] D. Masten, R. Hanson, C. Bowman, Journal of Physical Chemistry 94 (1990) 7119-7128.

[23] C. P. Robert, G. Casella, Monte Carlo Statistical Methods, Springer-Verlag, New York, 1999.

[24] W. Press, S. A. Teukolsky, W. Vetterling, B. Flannery, Numerical Recipes in C: The Art of Scientific Computing, 2nd Edition, Cambridge University Press, Cambridge, UK, 1992.

[25] D. A. Masten, A shock tube study of reactions in the $\mathrm{H}_{2} / \mathrm{O}_{2}$ mechanism, Ph.D. thesis, Stanford University, Stanford, CA (October 1990).

[26] C. Safta, H. Najm, O. Knio, Sandia Report SAND2011-3282, http://www.sandia.gov/tchem.

[27] S. D. Cohen, A. C. Hindmarsh, Comput. Phys. 10 (2) (1996) 138-143. 
a)

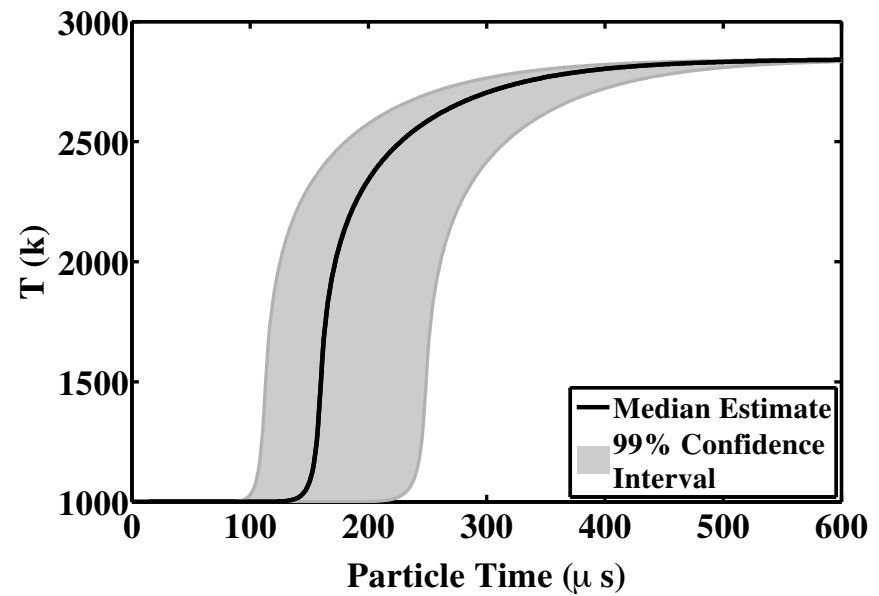

b)

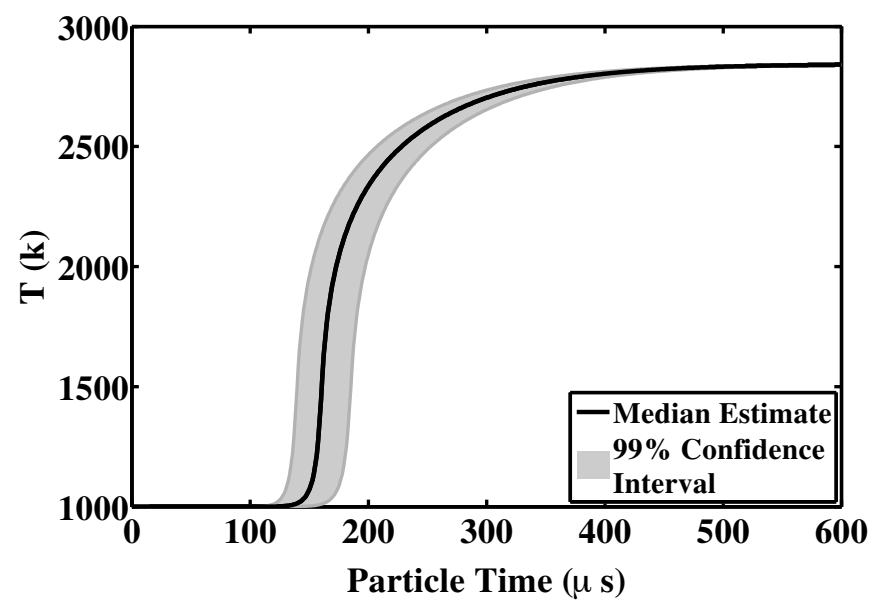

c)

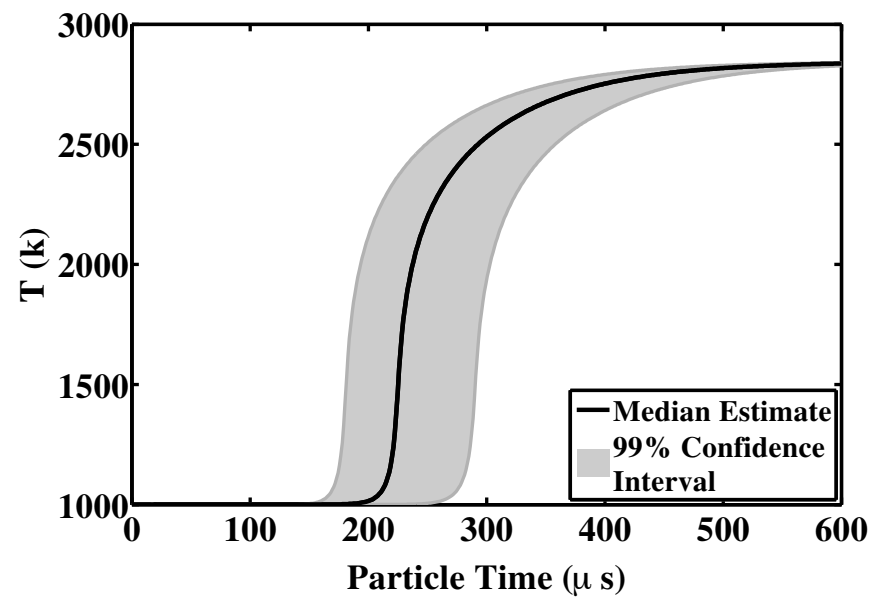

Fig. 6: Median temperature profiles and 99\% confidence interval for stoichiometric hydrogen-air autoignition at initial temperature of $1000 \mathrm{~K}$ and atmospheric pressure with Arrhenius parameters distributed according to cases (a) through (c). 


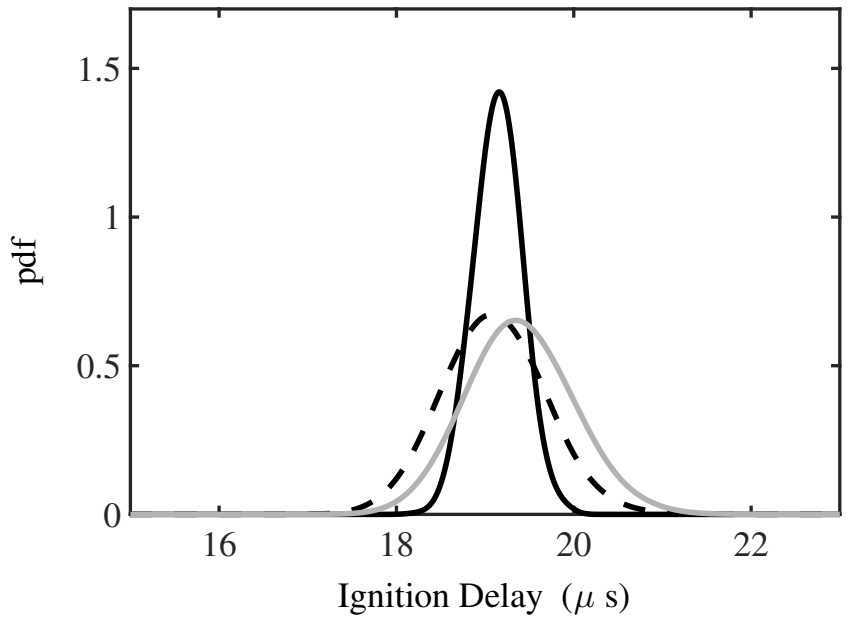

Fig. 7: Ignition delay time PDF for stoichiometric hydrogen-air auto-ignition at initial temperature of $1500 \mathrm{~K}$ and atmospheric pressure using Arrhenius parameters distributed according to case (a) in solid black curve, case (b) in dashed black curve, and case (c) in solid gray curve. 
a)

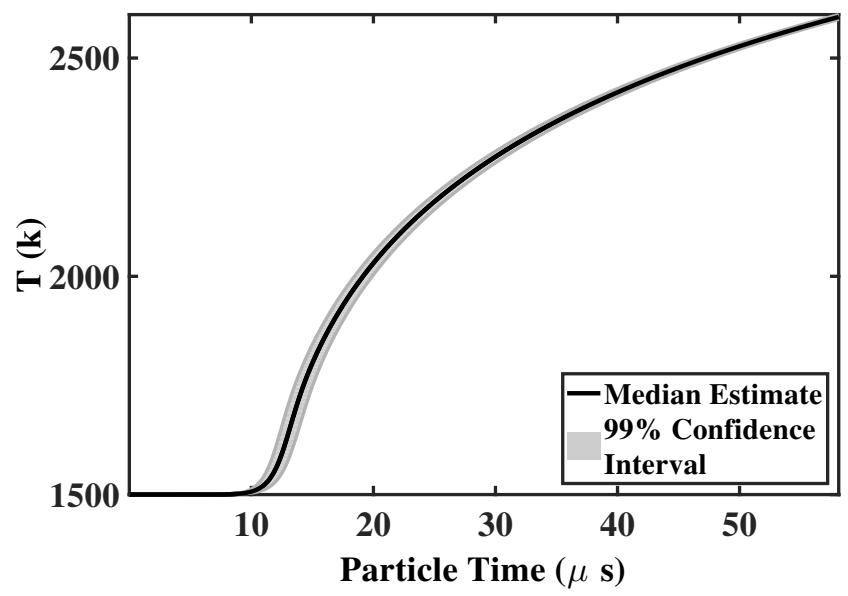

b)

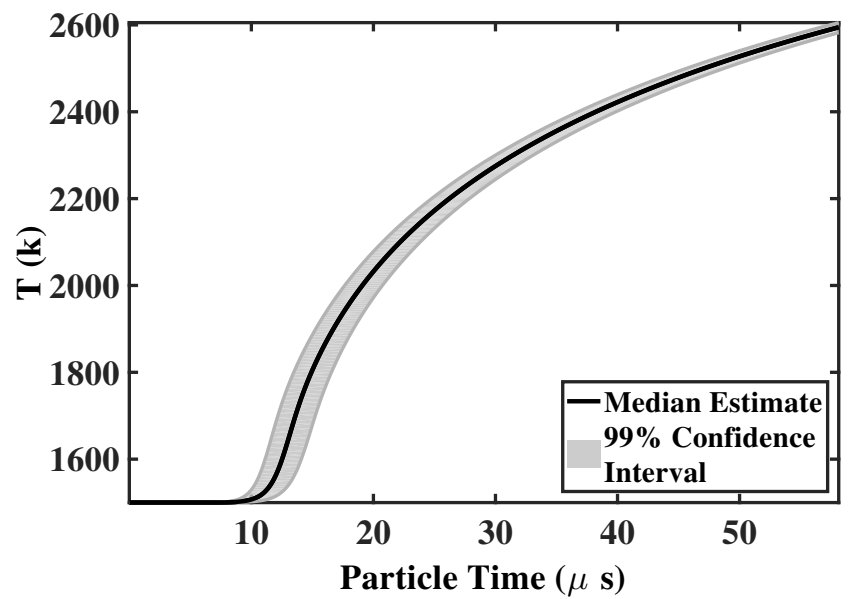

c)

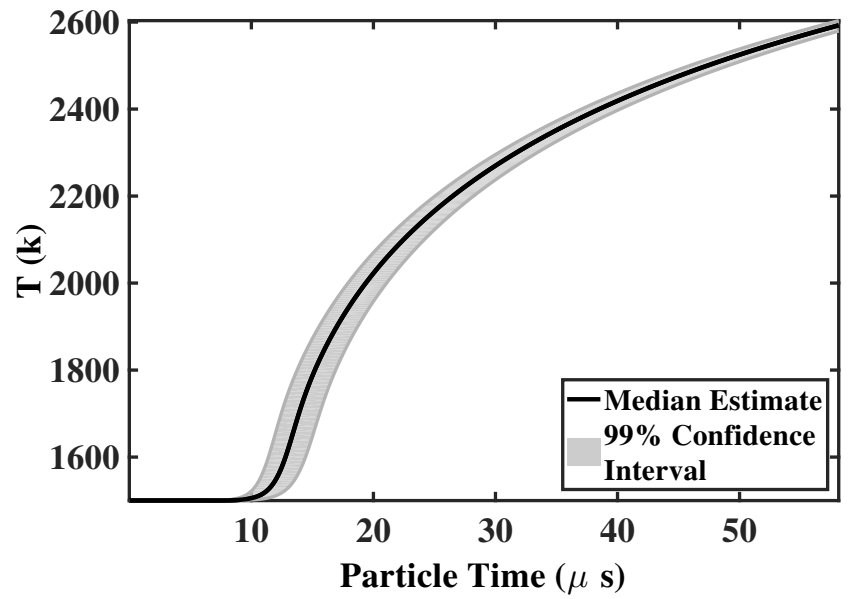

Fig. 8: Median temperature profiles and 99\% confidence interval for stoichiometric hydrogen-air autoignition at initial temperature of $1500 \mathrm{~K}$ and atmospheric pressure with Arrhenius parameters distributed according to cases (a) through (c). 